\title{
Nanomaterials for Drugs Delivery
}

\author{
Francisco Márquez ${ }^{1}$, Carmen Morant ${ }^{2}$ \\ ${ }^{1}$ Nanomaterials Research Group, School of Science and Technology, University of Turabo, Gurabo, USA \\ ${ }^{2}$ Department of Applied Physics, Universidad Autónoma de Madrid, Madrid, Spain \\ Email: fmarquez@suagm.edu
}

Received 1 May 2014; revised 1 June 2014; accepted 1 July 2014

Copyright (C) 2014 by authors and Scientific Research Publishing Inc.

This work is licensed under the Creative Commons Attribution International License (CC BY).

http://creativecommons.org/licenses/by/4.0/

(c) (7) Open Access

As expected for years, nanotechnology has revolutionized engineering, biology, chemistry, physics and medicine of today. These disciplines are evolving thanks to the ongoing development of new materials and applications. Nanomedicine, as application of nanotechnology in the field of health care, has undergone unprecedented development. Some of these changes have real applications as, for example, the use of nanoparticles in MRI imaging, in hyperthermia, in immunotherapy, or to improve the bioavailability of drugs, among others [1]-[3].

When a drug is administered to a patient, the blood distributes it throughout the body. In the case of very localized diseases (i.e. tumors), only a small fraction of the drug reaches the target. Chemotherapy is one of the most aggressive treatment options used in some types of cancer, and is usually administered intravenously. In this type of therapy, the drug circulates throughout the body, reaching and destroying healthy and cancerous tissues, producing side effects throughout the body, sometimes with serious consequences for the health of the patient (nephrotoxicity, cardiotoxicity, peripheral neuropathy, anemia, etc.). Among the many applications of nanotechnology, the fabrication of nanostructures capable of safely transporting these drugs is seen as a strategy for reducing these side effects. Nanoparticles are able to carry and release the drug in the right place and with the required dose, greatly reducing the problems associated with direct treatment with these drugs.

In recent years, there have been continuous improvements in the design and development of new tailor-made drug delivery systems [4], including hollow magnetic nanoparticles, liposomal structures, dendrimers, nanoporous silicon, etc. These structures can be obtained with different molecular weights (in the case of polymers), structures, shapes, and even with the appropriate functional groups for interaction at the desired positions. However, a great effort is still required to solve many of the current problems [5], including toxicity, aggregation, solubility and stability in the human body, physiological processes of elimination, identification of targets by highly specific receptors, controlled drug release over time, etc.

\section{References}

[1] Kim, J., Piao, Y. and Hyeon, T. (2009) Multifunctional Nanostructured Materials for Multimodal Imaging, and Simultaneous Imaging and Therapy. Chemical Society Reviews, 38, 372-390. http://dx.doi.org/10.1039/b709883a

[2] Barreto, J.A., O’Malley, W., Kubeil, M., et al. (2011) Nanomaterials: Applications in Cancer Imaging and Therapy. Advanced Materials, 23, H18-H40. http://dx.doi.org/10.1002/adma.201100140

[3] Smriti, M. and Bhaskar, S. (2014) Nanocarrier-Based Immunotherapy in Cancer Management and Research. ImmunoTargets and Therapy, 3, 121-134. 
[4] Li, Z., Barnes, J.C., Bosoy, A., et al. (2012) Mesoporous Silica Nanoparticles in Biomedical Applications. Chemical Society Reviews, 41, 2590-2605. http://dx.doi.org/10.1039/c1cs15246g

[5] Dobrovolskaia, M.A. and Mcneil, S.E. (2007) Immunological Properties of Engineered Nanomaterials. Nature Nanotechnology, 2, 469-478. http://dx.doi.org/10.1038/nnano.2007.223 